\title{
Multi-staged granular beds applied to the filtration of ultrafine particles : an optimization of collector diameters
}

\author{
Augustin CHARVET ${ }^{1}$, Loïc WINGERT ${ }^{1-2}$, Nathalie BARDIN-MONNIER ${ }^{1}$, Stéphanie PACAULT ${ }^{1}$, Frantz \\ FOURNIER $^{1}$, Denis BEMER ${ }^{2}$, Dominique THOMAS ${ }^{1}$
}

\author{
${ }^{1}$ Laboratoire Réactions et Génie des Procédés, Université de Lorraine, CNRS, LRGP, F-54000 Nancy, France \\ ${ }^{2}$ Institut National de Recherche et de Sécurité, Vandœuvre-lès-Nancy, F-54519, France
}

Corresponding author: augustin.charvet@univ-lorraine.fr; Tel: +33(0) 372743725

\begin{abstract}
$\underline{\text { Abstract }}$
Granular beds exhibit interesting performances, in terms of ultrafine particle collection efficiency, hot gas cleaning, low cost and robustness. Nevertheless, some authors highlighted that ultrafine particle deposit is essentially located in the first layers of the granular bed. Thus, only a small depth is useful thereby reducing both the operation time between two uncloggings and the process yield. Our improvement approach consists in reducing the pressure drop increase while maintaining high collection efficiency by using a granular bed presenting a collector diameter gradient. Performances of this improved granular bed and of a conventional one were compared during their clogging with particles generated by thermal spraying. Since the collection efficiency is a decreasing function of the collector size, this improved configuration permits to firstly clog the downstream layers of the granular bed. Although there is a $20 \%$ decrease of the initial efficiency for this new type of bed compared to the conventional one, this gap is rapidly reduced during the clogging process. At the same time, the results highlight a lower pressure drop increase of the modified granular bed. A predictive clogging model was also developed in order to find an optimized configuration of collector diameter to ensure a contribution of the whole granular bed depth.
\end{abstract}

\section{$\underline{\text { Keywords }}$}

Granular bed, Optimization, Performances, Nanoparticles

\section{Introduction}

A large amount of ultrafine particles are generated by manufacturing processes such as surface processing (thermal spraying), machining (drilling, grinding...), combustion, welding and cutting of metals, ... The development of suitable protection systems therefore appears crucial in order to protect the persons and the environment. The most efficient and widely used de-dusting methods for separating the particles from a carrier fluid are currently pleated fiber filters. These fibrous media are initially very efficient but generate a strong increase in pressure drop because of a quick and sometimes irreversible clogging as well as an extra cost resulting from their replacement. Following these observations, we propose to investigate the potential of other methods that could be applied to ultrafine particle collection. Among them, granular beds exhibit interesting performances, as far as ultrafine particle collection efficiency is concerned, and a higher resistance to mechanical and thermal stresses comparatively to fibrous filters. Moreover, another advantage of granular bed filters is their cleanability and reusability in opposition to the difficulty of unclogging the particles deposited on fibrous filters. This specificity is even more important because the reusability of the granules reduces the variability of pressure drop and collection efficiency in research investigations and of process performances in industrial applications.

Aerosol collection in granular beds has been the subject of many theoretical and experimental studies since 1970 and a thorough review of those can be found in Tien's book [1]. Experimental studies focused mainly on the overall performances (pressure drop and collection efficiency) of a granular filter by changing the filter construction parameters, superficial gas velocity [2], temperature [3], bed depth and porosity, granular collector characteristics $[4], \ldots$ 
Results highlight that an optimization of the granular bed parameters (collector diameter, granular bed thickness, face velocity of the effluent, magnetic stabilization, etc.) could bring this technique to become a viable filtration method for ultrafine particles. However, all of these studies were limited to the characterization of granular beds in their initial state and the evolution of their performances during the particle loading, i.e. during the accumulation of particles within the bed, is much less examined. Moreover, these studies were dedicated to the performances of granular beds towards micronic and submicronic particles and consequently results do not guarantee the performances of such granular bed filters towards ultrafine particles, nanostructured or not.

Furthermore, Bémer et al. [5] showed that ultrafine particle deposit is essentially located in the first layers of a granular bed. Thus, only a small depth is useful thereby reducing both operation time of granular beds between two uncloggings and the process yield. In order to optimize the performance of granular beds, Thivel et al. [6] emphasized that it may be interesting to use a magnetically stabilized bed because it combines high dust collection efficiencies with a reduced energy cost. In more recent studies, special attention has been devoted to the regeneration of the filter granules. In this way, moving granular bed filters, in countercurrent [7-8] or cross-currents configurations [9-11] use a continuous flow of fresh collectors to limit the formation of a filtration cake and maintain a low and constant pressure drop, without the need for a periodic unclogging. However, moving granular beds have some drawbacks related to the transport of collectors and present a lower collection efficiency as dust cake is continuously re-entrained. Moreover, operating principle of panel beds is somewhat different and based on a periodic renewal of the granular bed surface. Hence, these panel beds mimic fabric filters by taking advantage of the high collection efficiency of a particle cake. To decrease the pressure drop, a cyclical offline puff-back induces the fall of this filtration cake and of the first layers of collectors which are replaced with fresh ones [1215].

Our optimization approach is quite different and consists in increasing the time between two unclogging operations by reducing the pressure drop increase while maintaining a high collection efficiency. Tian et al. [16] applied this approach, using a dual-layer granular bed for the filtration of micron-sized ash particles. Nevertheless, the very thick, and consequently very efficient, upper stage implemented in this study reduces the interest of the lower stage. Indeed, during the clogging, the deposit is mainly located in the first layers of collectors which render the remaining thickness of the upper stage unnecessary. With this in mind, a granular bed with a gradient of collector sizes was proposed. From a theoretical standpoint, as the collection efficiency is a decreasing function of the collector size in a diffusional regime, a granular bed with decreasing collector sizes in the depth should permit to firstly clog the lower stages of the granular bed and consequently delay the pressure drop increase (cf. Figure 1). To limit the experimental constraints, we chose to study the simplest "gradient" granular bed, consisting in only three stages. Each stage is composed of beads satisfying the condition that a given stage must be filled with smaller beads than those of the previous upper stage. Moreover, it was decided to limit the thickness of each stage given that only the first layers of collectors are effective in a conventional granular bed, i.e. a granular bed composed of beads with given diameter.

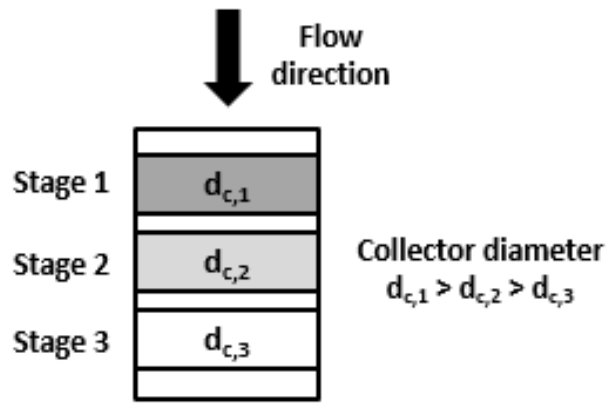

Figure 1: Schematic view of the multi-staged granular bed 


\section{Experimental approach}

Experiments were achieved using a three-stage $40 \mathrm{~mm}$-diameter column filled with stainless steel beads. The performances of a multi-staged granular bed composed of $1 \mathrm{~mm}$-diameter beads in the first stage, $0.8 \mathrm{~mm}$ in the second and $0.5 \mathrm{~mm}$ in the third were compared to those of a conventional one, i.e. a granular bed with bead diameter of $0.5 \mathrm{~mm}$ in the three stages. For the reasons mentioned above, the depth of each granular bed stage was chosen to approximately $11 \mathrm{~mm}$. To ensure that the porosity was as close as possible to the one of a random close packing of spheres, the granular beds in each stage were packed by applying vibrations until reaching a relative gap lower than 5\% between the experimental pressure drop and the one predicted by the Kozeny-Carman's law. Static differential pressure sensors were mounted upstream and downstream of each stage allowing the monitoring of the pressure drop evolution during the clogging.

The particles, composed of $85 \%$ of zinc and $15 \%$ of aluminium, were generated by thermal spraying of metal using an electric arc gun (Margarido ${ }^{\circledR}$ M25). These nanoparticles were then directed towards a sharp cut cyclone (BGI ${ }^{\circledR}$ SCC 1.829) to collect the micron-sized fraction of the produced aerosol and clog the granular bed only with the fine fraction. The particle size distribution of the resulting particles of $\mathrm{Zn}-\mathrm{Al}$ was measured with a Scanning Mobility Particle Sizer (SMPS 5.400 Grimm ${ }^{\circledR}$ ) and presented a median number diameter, expressed in equivalent electric mobility, of $78.3 \mathrm{~nm}$ with a geometric standard deviation of 1.6. The total number concentration was approximately $2.10^{8}$ particles $/ \mathrm{cm}^{3}$. The total mass concentration, measured by a gravimetric method after sampling on glass fiber filters, was close to $72.5 \mathrm{mg} / \mathrm{m}^{3}$. The air flowrate was controlled by a sonic nozzle and measured by a mass flowmeter (TSI ${ }^{\circledR} 4100$ ). It was set to $15 \mathrm{~L} / \mathrm{min}$ corresponding to a superficial velocity of $0.2 \mathrm{~m} / \mathrm{s}$.

The particle number size distributions were measured upstream and downstream of each stage using a Nanoscan analyzer (SMPS Model 3910, TSI ${ }^{\circledR}$ ) after a dilution stage (VKL100, Palas ${ }^{\circledR}$ ). These sequential measurements, called cycles, consisted of three scans of one minute upstream of each stage followed by three scans downstream. The three scans of a same sampling cycle were averaged to obtain the mean upstream and downstream fractional number concentrations $\left(\mathrm{C}_{\mathrm{n}, \mathrm{i}}\right)$ which were converted according to equation 1 into fractional mass concentrations $\left(\mathrm{C}_{\mathrm{m}, \mathrm{i}}\right)$. It was therefore possible to obtain the fractional mass collection efficiencies and finally the total collected mass collected at each time step for each stage.

$$
\begin{gathered}
C_{m, i}=\frac{\pi \cdot \rho_{P}}{6} \cdot C_{n, i} \cdot d_{V, i}^{3}=\frac{\pi \cdot \rho_{e, i}}{6} \cdot C_{n, i} \cdot d_{m, i}^{3} \\
\rho_{e, i}=40.24 \cdot d_{m, i}^{-0.91}
\end{gathered}
$$

where $\rho_{\mathrm{p}}$ is the density of the raw material $\left(5740 \mathrm{~kg} / \mathrm{m}^{3}\right.$ for the $\left.\mathrm{Zn}-\mathrm{Al}\right), \mathrm{d}_{\mathrm{v}, \mathrm{i}}$ the volume equivalent diameter, $\mathrm{d}_{\mathrm{m}, \mathrm{i}}$ the electric mobility equivalent diameter and $\mathrm{C}_{\mathrm{n}, \mathrm{i}}$ the measured fractional number concentration and $\rho_{\mathrm{e}, \mathrm{i}}$ the effective density of the $\mathrm{Zn}$-Al agglomerates. The effective density, defined as the ratio of aggregate mass to equivalent mobility volume, allows converting the volume equivalent diameter into an electric mobility equivalent diameter and consequently calculating the fractional mass concentrations. It should be noted that in the equation 2, providing the relationship between the effective density of the agglomerates and the electric mobility equivalent diameter, the diameter is expressed in $\mathrm{nm}$ and the effective density in $\mathrm{g} / \mathrm{cm}^{3}$ [17]. Furthermore, this equation is only applicable for electric mobility equivalent diameters higher than the diameter of the primary particles constituting the agglomerates, i.e. $9 \mathrm{~nm}$ for $\mathrm{Zn}$-Al primary particles ; otherwise the effective density will become higher than the raw particle material. 

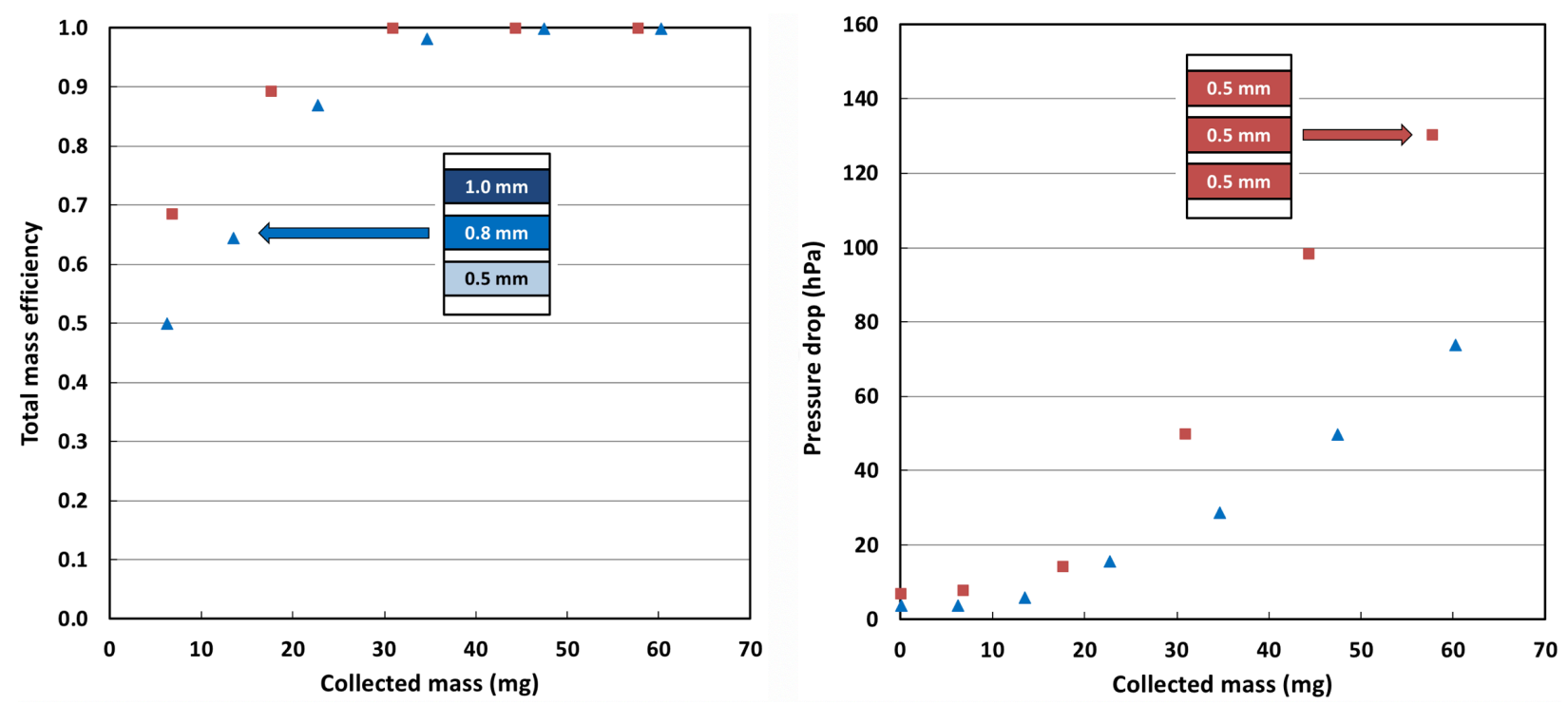

Figure 2: Total mass efficiency and pressure drop evolutions of conventional and multi-staged granular beds

The performances of the two configurations were compared in terms of global mass efficiency and pressure drop as function of the collected mass (cf. Figure 2). As collection efficiency is a decreasing function of the collector size, the results show a lower initial efficiency for the multi-staged granular bed compared to the conventional one. But, this gap is rapidly reduced during the clogging process. At the same time, a lower pressure drop increase of the multi-staged granular bed is shown by the results. Indeed, the pressure drop is quite three times lower than the one of the conventional bed when the total mass efficiency reaches a value close to unity.
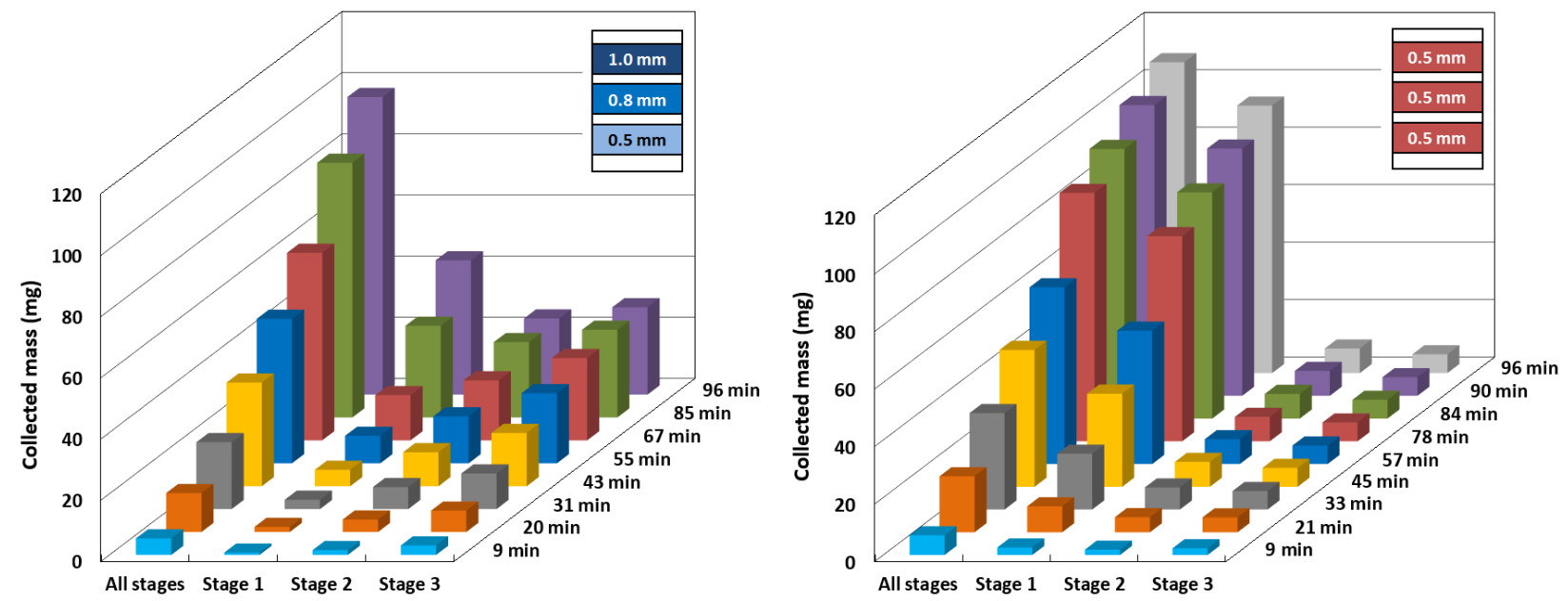

Figure 3: Mass of particle collected in each of the three stages during the clogging of a multi-staged (left) and a conventional (right) granular bed

The plot of the mass collected according to time and stage confirms that the deposition in a conventional granular bed mainly occurs in the first stage (cf. Figure 3). In the case of the multi-staged granular bed, the mass collected seems to be more homogenously distributed. Indeed, this figure clearly shows that the use of a gradient of bead diameter permitted to firstly clog the downstream layers of the granular bed, i.e. the third stage of the multi-staged column. According to these experimental results, the improvement of the granular bed filtration by means of a collector diameter gradient appears quite promising in order to extend the operation time between two uncloggings. Nevertheless, it was noticed that the first stage of this configuration becomes efficient sooner than expected and consequently that the second and third stages did not contribute enough to the particle retention. This combination of bead diameters $(1 \mathrm{~mm} / 0.8 \mathrm{~mm} / 0.5 \mathrm{~mm})$ is consequently not optimal. 


\section{Modeling approach}

As a result of these experimental observations, it was decided to find an optimized configuration of bead diameters so that the whole granular bed depth contributes to the filtration. As an experimental approach would be too timeconsuming, we shifted towards a modeling approach using a previously developed model predicting the temporal evolution of pressure drop and collection efficiency and determining the location of the mass collected in the granular bed depth, for given operating conditions (filtration velocity, pressure, temperature ...) and given granular bed parameters (bead size, thickness ...).

This model, developed for conventional granular beds, has been modified to evaluate the performances of multistaged granular beds. These adjustments only consist of a repetition of the original model for each stage of the granular bed placed in series. In this way, the output data of the first stage become the input data of the second stage and so on. A brief description of this clogging model is given below. For more detailed information, readers can refer to [18].

In the model, the deposit of ultrafine particles is assimilated to a uniform and spherical shell all over the collector's surface. This assumption has been used in previous studies $[19,20]$ and validated by microscopic observations [21]. According to this uniformity feature of the deposit, the principle is based on the calculation of equivalent collector diameters depending on the collected mass of particles and to implement them in initial pressure drop (equation 3) and collection efficiency (equation 4) models.

$$
\begin{gathered}
\Delta \mathrm{P}=36 \cdot \mathrm{h}_{\mathrm{k}} \cdot \mu \cdot \mathrm{U} \cdot\left(\frac{\left(1-\epsilon_{\mathrm{GB}}\right)^{2}}{\epsilon_{\mathrm{GB}}^{3}}\right) \cdot\left(\frac{\mathrm{z}_{\mathrm{GB}}}{\mathrm{d}_{\mathrm{c}, \mathrm{eq}}^{2}}\right) \\
\mathrm{E}_{\mathrm{GB}}=1-\exp \left(-\frac{3}{2} \cdot\left(\frac{1-\epsilon_{\mathrm{GB}}}{\mathrm{d}_{\mathrm{c}, \mathrm{eq}}}\right) \cdot \mathrm{z}_{\mathrm{GB}} \cdot \eta\left(\mathrm{d}_{\mathrm{c}, \mathrm{eq}}\right)\right)
\end{gathered}
$$

with $h_{k}$ the Kozeny-Carman constant, $\mu$ the air viscosity, $U$ the superficial velocity, $\mathrm{z}_{\mathrm{GB}}$ the granular bed depth, $d_{c, \text { eq }}$ the equivalent collector diameter, $\epsilon_{\mathrm{GB}}$ the granular bed porosity and $\eta\left(\mathrm{d}_{\mathrm{c}, \mathrm{eq}}\right)$ the single collector collection efficiency depending on the equivalent collector diameter.

Results of clogging experiments have highlighted that the deep bed filtration can be split in two phases requiring the use of two different kinds of collector equivalent diameter. During the first phase, called phase A, the pressure drop and the efficiency remain approximately constant indicating an insignificant change in the granular beds structure. The collector diameter is assumed to increase slightly without any blocking of the pore spaces. Thus, during the phase $\mathrm{A}$, the equivalent collector diameter is considered as the one of the sphere having the same volume as a collector and its deposit layer and can be expressed as:

$$
d_{c, e q, A}=\left(d_{c}^{3}+\frac{6 \cdot m_{p, c, A}}{\pi \cdot \rho_{p} \cdot\left(1-\epsilon_{d}\right)}\right)^{\frac{1}{3}}
$$

with $d_{c, e q, A}$ the instantaneous equivalent collector diameter during the phase $A, d_{c}$ the initial collector diameter, $\mathrm{m}_{\mathrm{p}, \mathrm{c}, \mathrm{A}}$ the instantaneous mass collected per collector during the phase $\mathrm{A}, \rho_{\mathrm{p}}$ the density of the raw material and $\varepsilon_{\mathrm{d}}$ the deposit's porosity.

The second clogging phase, called phase B, is characterized by a sharp increase of the pressure drop and of the collection efficiency. This performance evolution is assigned to an increase of the collector specific area because of the deposit accumulation. Consequently, during the phase B, the equivalent collector diameter is defined as the one of the sphere having the same specific area as a collector and its deposit layer:

$$
d_{c, e q, B}=\left(\frac{\pi \cdot d_{c, e q, A}^{3} \cdot \rho_{p} \cdot\left(1-\epsilon_{d}\right) \cdot d_{v, 50}+6 \cdot d_{v, 50} \cdot m_{p, c, B}}{\pi \cdot d_{c, e q, A}^{2} \cdot \rho_{p} \cdot\left(1-\epsilon_{d}\right) \cdot d_{v, 50}+4 \cdot\left(1-\epsilon_{d}\right) \cdot m_{p, c, B}}\right)
$$

with $d_{v, 50}$ the median volume equivalent diameter of the collected particles and $m_{p, c, B}$ the instantaneous mass collected per collector during the phase B. 
As the equivalent diameters are calculated at each increment of collected mass, this model allows determining the temporal performances of the granular bed. Concerning the spatial arrangement of the deposit in the granular bed depth, it is made possible by means of its discretization in layers.

\section{Multi-criteria optimization}

All the operating conditions previously described are maintained (filtration velocity, thickness of each stage ...) and the model was implemented for a set of possible combinations of collector diameters. The collector size ranged from $0.2 \mathrm{~mm}$ to $1.6 \mathrm{~mm}$ by increment of $0.1 \mathrm{~mm}$. The multi-staged granular beds simulated had to satisfy the condition that a given stage must be filled with smaller beads than those of the previous upper stage.

These settings led to 455 combinations of collector diameters. The performances of these 455 multi-staged granular beds were compared with each other based on three criteria:

- The retention capacity, i.e. the total mass collected per volume unit during the time necessary to reach an efficiency of the first stage close to $99 \%$; it is considered that above this value, the particles will no more be collected in the granular bed depth but at the surface of the first stage. In this way, industrially, the beginning of the surface filtration will correspond to a need of an unclogging operation.

- The mean mass efficiency during this same clogging duration, i.e. the ratio of the total collected mass to the total generated mass;

- The reciprocal of the pressure drop.

To obtain the best performances, each of these criteria should be maximized. As the optimum of each criterion is obtained for different combinations and as each criterion has not the same importance, it is difficult to identify the best trade-off solution. A classical approach consists in merging all criteria in a single one. This aggregation approach induces some subjectivity as it is necessary to attribute a relative weighting to each criterion.

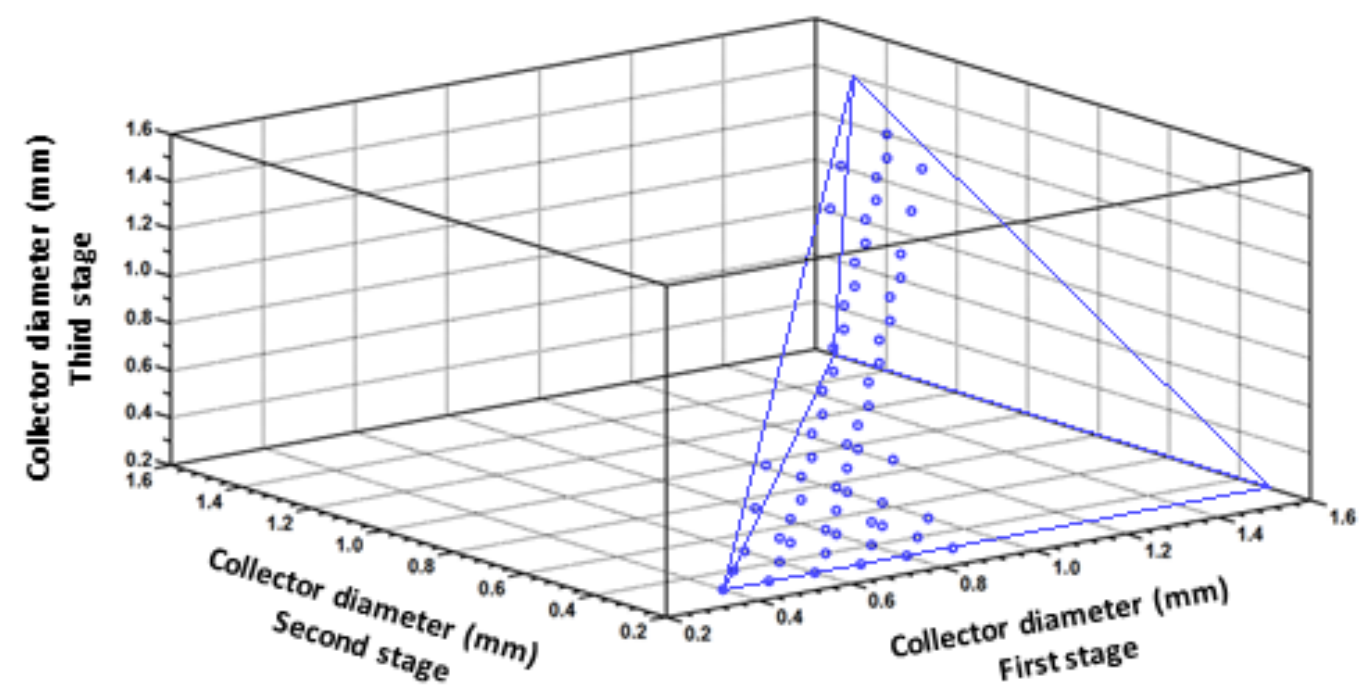

Figure 4: Result of the Pareto domination analysis (the points represent the 61 trade-off solutions among the 455 possible combinations within the tetrahedron)

We chose another approach in order to reduce this subjectivity. It is based on an optimization in a first step and introducing preferences, i.e. subjectivity, as late as possible in the decision process in order to identify the whole set of trade-off solutions. To identify this set of trade-off solutions, the Pareto's domination concept has been applied [22]. This analysis relies on a comparison of all the combinations by pairs in order to determine if a given combination is dominated by another one.

Considering two combinations $\mathrm{X}$ and $\mathrm{Y}, \mathrm{X}$ is regarded as not dominated by $\mathrm{Y}$ if:

- All the criteria of $X$ are higher than or equal to all the equivalent criteria of $Y$;

- At least one criterion of $X$ is strictly better than the equivalent criterion of $Y$. 
During this analysis by pairs, a range of indifference has been added in order to take into account the model uncertainty. This range is defined as the difference between two values of the same criterion that is not significant enough to consider a value as preferred over another one.

By selecting only the never dominated configurations, this Pareto's domination analysis allows considerably reducing the number of possible combinations (61/455), without introducing subjective weightings between the three chosen criteria (cf. Figure 4). To go further, subjective weightings have to be introduced to define a ranking between these 61 selected combinations. A multi-criteria optimization technique, called rough set method, has been applied [23-24]. This method consists in the selection of a small sample of seven combinations, being broadly representative of those retained after the multi-criteria analysis described previously. These seven chosen combinations have to be manually ranked, from the most to the least preferred, by a human expert who has a deep knowledge of the process.

Then, the combinations of the sample are compared by pairs for each criterion in order to establish a set of rules based on the expert's ranking. If one criterion of a preferred combination is better than the same criterion of a less preferred one, the value of 1 is affected to this criterion. Otherwise, it obtains the value of 0 . From these comparisons result binary rules of preference. For example, the preference rule $1 / 0 / 1$ is obtained if the first criterion of a combination is better, the second criterion worse and the third one better than the same criteria of a less preferred combination. Repeating this step, from the less to the most preferred combination, permits to get the binary rules of non-preference. The binary rules appearing as both a preference and a non-preference rule are eliminated.

The remaining ones are then applied to the 61 previously selected combinations by comparing them by pairs. If a comparison of two combinations corresponds to a rule of preference, the ranking score of the first combination is incremented by one unit and the score of the second combination decremented by one unit. If the comparison leads to a non-preference rule, one counts -1 point for the first combination and 1 for the second one. When the comparison matches neither a preference nor a non-preference rule, the ranking scores of the two combinations remain unaltered. This allows ranking all the combinations according to their final score, the highest representing the one that has to be finally chosen.

After the two optimization phases, consisting in the Pareto's domination analysis and the Rough set method, the optimal trade-off solution obtained is a combination with small bead diameters $(0.6 / 0.4 / 0.2 \mathrm{~mm})$. The results are quite similar whatever the expert chosen. The modeled performances of this optimal multi-staged granular bed have been compared to the ones of the two combinations experimentally tested and to the ones of the extremum combination, i.e. constituted of the smaller collectors.
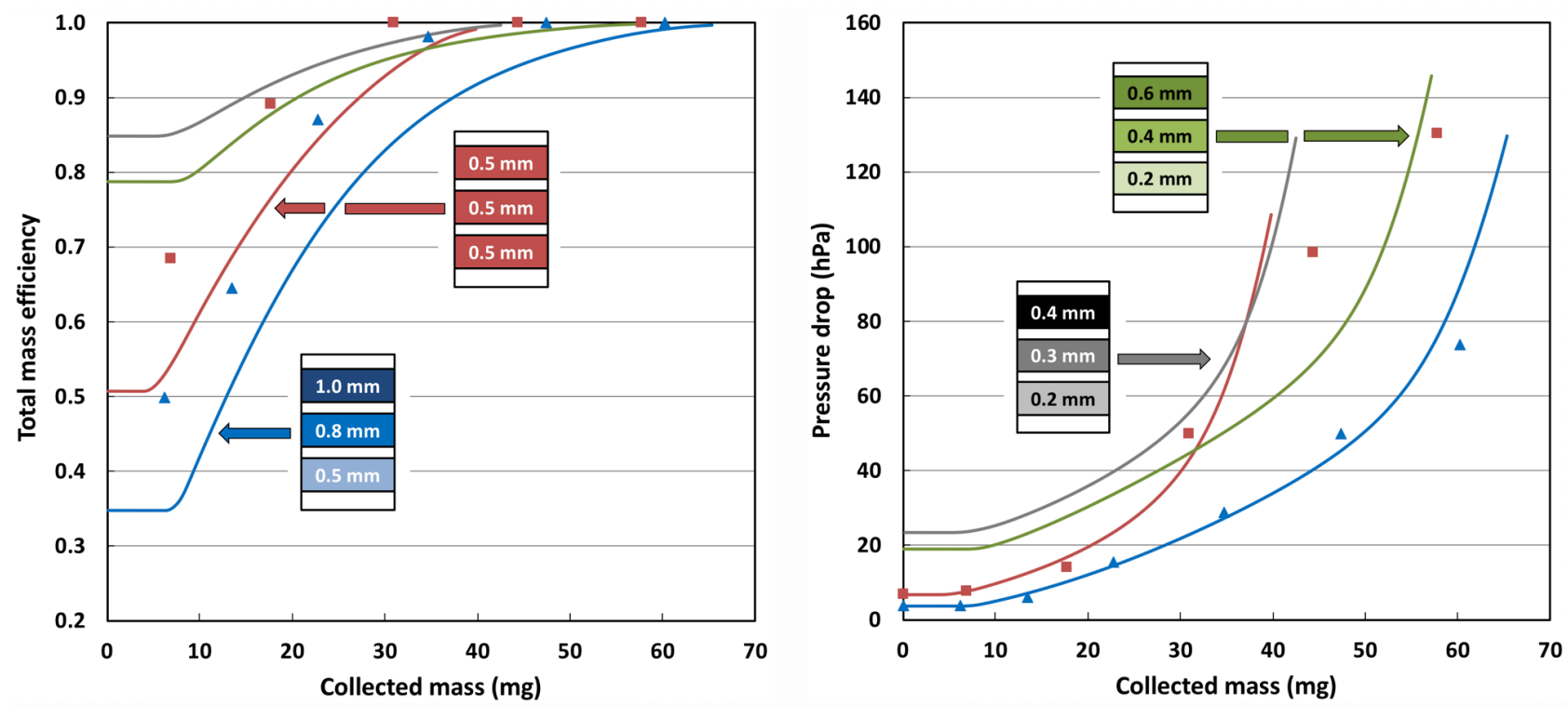

Figure 5: Efficiency and pressure drop evolutions during the clogging of multi-staged granular beds with various combinations of collector diameters 
Figure 5 (left) represents the efficiencies modeled for each combination as a function of the collected mass. As the collection efficiency is a decreasing function of the collector diameter, the modeled efficiencies are all the more high that the combination is constituted of small beads. Furthermore, for the combinations $1.0 / 0.8 / 0.5 \mathrm{~mm}$ and 0.5/0.5/0.5 mm, we can notice a good agreement between experimental data and model-based predictions even if there is a slight underestimation of the collection efficiency by the model. This difference is mainly caused by a lack of precision in the correlation used in the model for the calculation of initial efficiency by Brownian diffusion [18]. As the Neale and Nader hydrodynamic factor implemented in the model tends to slightly underestimate the efficiency of a conventional granular bed, this trend is even more marked for three granular beds in series.

At the same time, the model satisfactorily predicts the experimental pressure drop evolution (Figure 5, right). The combination 1.0/0.8/0.5 mm presents the lower energy expenditure among the tested multi-staged granular beds but also the lower mass efficiency. The trade-off solution appears to be the combination $0.6 / 0.4 / 0.2 \mathrm{~mm}$ as the mass efficiency is higher than $80 \%$ and the pressure drop increase relatively slow. The model also allows a fairly good prediction of the clogging profile as modeled and experimental mass fractions per stage follow the same trend (cf. Figure 6). As previously mentioned, the experimental fractions of mass collected are calculated from the upstream and downstream fractional mass concentrations deduced from the Nanoscan data. Figures 6A and 6D highlight that a gradient of collector diameters allows clogging firstly the deepest stage contrary to a conventional granular bed in which the particle deposition mainly occurs in the upper stage (cf. Figure 6B). After a long period of particle loading, the particles are more homogeneously distributed in the depth of the bed. Contrary to a conventional granular bed in which particles are collected in the first layers, the particle mass is retained in a higher volume and consequently leads to a lower pressure drop increase. The results obtained for the combination 0.4/0.3/0.2 mm show that if collector diameters in two consecutive stages are too close to each other, the multistaged granular bed loses all of its advantages as particle deposition quickly becomes mainly located in this first stage rending the two other stages unnecessary, as in a conventional granular bed (Figure 6C). This is all the more emphasized since the upper stage is efficient. Thus, the difference between each initial collection efficiency should be sufficient to preferentially collect the particles into the lower stage.

\section{Conclusion}

According to experimental results, using a multi-staged granular bed with a collector diameter gradient appeared promising in order to obtain a more homogeneous deposit and extend the operation time between two uncloggings. The choice of collector diameters is essential to reach simultaneously a high initial efficiency and a more homogeneous deposit, i.e. a delayed pressure drop increase. A predictive clogging model was also developed in order to simulate the temporal performance evolution of a set of possible combinations of collector diameter. Thereafter, a multi-criteria optimization was led and permitted to find an optimized configuration of bead diameters $(0.6 / 0.4 / 0.2 \mathrm{~mm})$ so that the whole granular bed depth contributes to the filtration. 

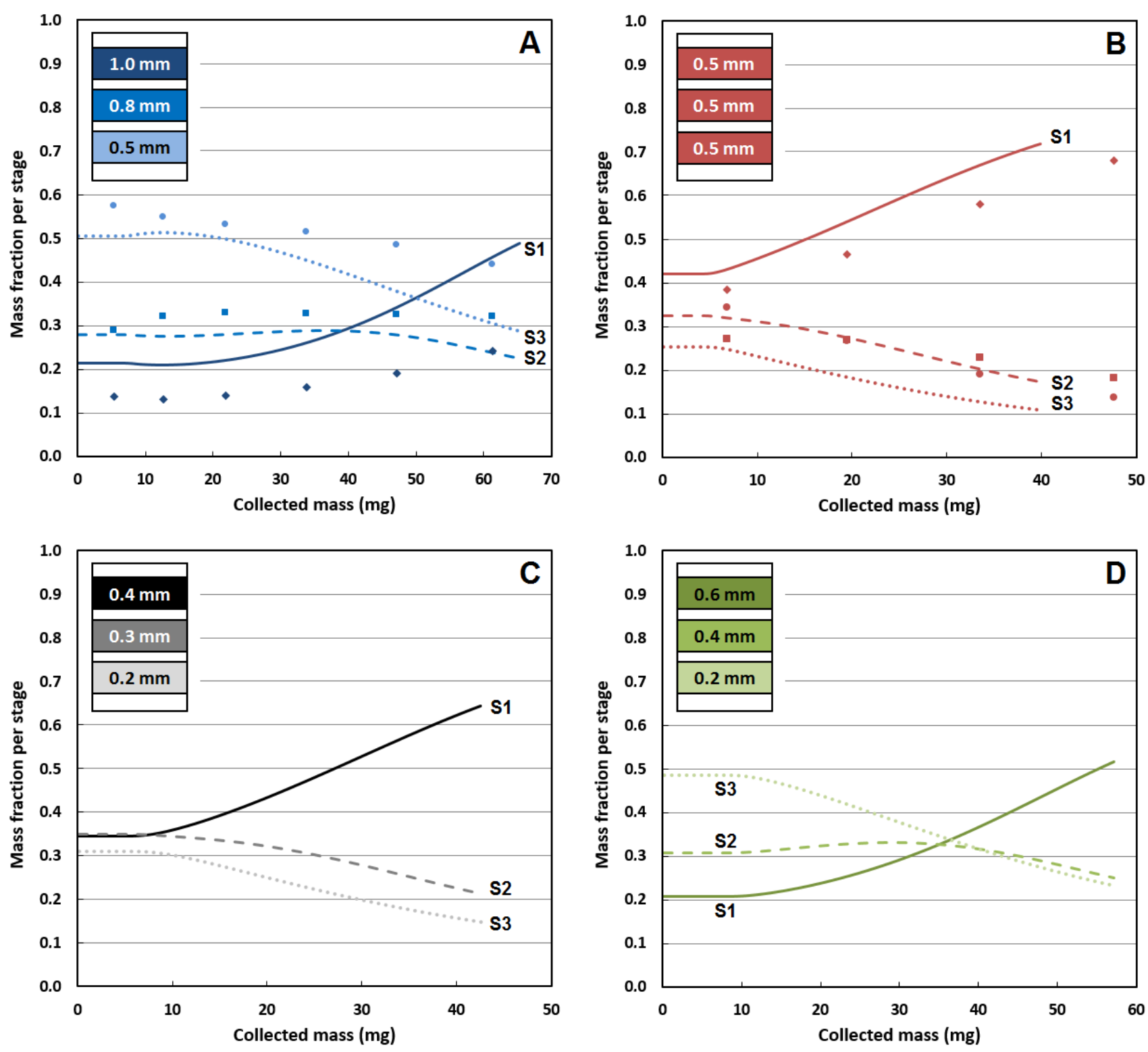

Figure 6: Mass fraction collected in each stage during the clogging of granular beds with various combinations of collector diameters. Solid, broken and dotted lines correspond to the modelling results and diamonds, squares and circles to experimental results for the first, second and third stages, respectively

\section{$\underline{\text { References }}$}

[1] C. Tien, Granular Filtration of Aerosols and Hydrosols, $1^{\text {st }}$ ed., Butterworths, London, 1989.

[2] J. Tsubaki, C. Tien, Gas filtration in granular moving beds - an experimental study, Canadian Journal of Chemical Engineering 66 (1988) 271-275.

[3] W. Peukert, F. Löffler, Influence of temperature on particle separation in granular bed filters, Powder Technology 68 (1991) 263-270.

[4] S.C. Saxena, R.F. Henry, W.F. Podolski, Particulate removal from high-temperature, high-pressure combustion gases, Progress in Energy and Combustion Science 11 (1985) 193-251.

[5] D. Bémer, I. Subra, A. Morele, A. Charvet, D. Thomas, Experimental study of granular bed filtration of ultrafine particles emitted by a thermal spraying process, Journal of Aerosol Science 63 (2013) 25-37. 
[6] P-X. Thivel, Y. Gonthier, P. Boldo, A. Bernis, Magnetically stabilized fluidization of a mixture of magnetic and non-magnetic particles in a transverse magnetic field, Powder Technology 139 (2004) 252-257.

[7] R.C. Brown, H. Shi, G. Colver, S.C. Soo. Similitude study of a moving bed granular bed, Powder Technology 138 (2003) 201-210.

[8] I.A. El-Hedok, L. Whitmer, R.C. Brown, The influence of granular flow rate on the performance of a moving bed granular filter, Powder Technology 214 (2011) 69-76.

[9] Y.S. Chen, S.S. Hsiau, S.C. Lai, Y.P. Chyou, H.Y. Li, C.J. Hsu, Filtration of dust particulates with a moving granular bed filter, Journal of Hazardous Materials, 171 (2009) 987-994.

[10] Y.S. Chen, S.S. Hsiau, J. Smid, J. Wu, S. Ma, Removal of dust particles from fuel gas using a moving granular bed filter, Fuel 182 (2016) 174-187.

[11] S.S. Hsiau, J. Smid, S.A. Tsai, C.C. Tzeng, Y.J. Yu, Flow of filter granules in moving granular beds with louvers and sublouvers, Chemical Engineering and Processing: Process Intensification 47 (2008) 2084-2097.

[12] K.C. Lee, R. Pfeffer, A.M. Squires, Granular-bed filtration assisted by filter-cake formation 1. Exploiting a new mode of soil failure for renewal of filtration surfaces in a panel bed, Powder Technology 155 (2005) 5-16.

[13] I. Rodon, K.C. Lee, R. Pfeffer, A.M. Squires, O.K. Sonju, Granular-bed filtration assisted by filter-cake formation 2. The panel bed gas filter with puffback renewal of gas-entry surfaces, Powder Technology 155 (2005) 52-61.

[14] M.S. Wu, K.C. Lee, R. Pfeffer, A.M. Squires, Granular-bed filtration assisted by filter-cake formation 3. Penetration of filter cakes by a monodisperse aerosol, Powder Technology 155 (2005) 62-73.

[15] A.M. Squires, Granular-bed filtration assisted by filter-cake formation 4. Advanced designs for panel-bed filtration and gas treating, Powder Technology 155 (2005) 74-84.

[16] S.R. Tian, G.H. Yang, Z. Li, K.Y. Shi, G.Z. Ding, Z.F. Hu, Cascade filtration properties of a dual-layer granular bed filter, Powder Technology 301 (2016) 545-556.

[17] A. Charvet, S. Bau, D. Bémer, D. Thomas, On the Importance of Density in ELPI Data Post-Treatment, Aerosol Science and Technology 49 (2015) 1263-1270.

[18] L. Wingert, N. Bardin-Monnier, A. Charvet, D. Bémer, D. Thomas, Modeling of the deep granular bed clogging by nanoparticles, Separation and Purification Technology 176 (2017) 384-394.

[19] C. Tien, R.M. Turian, H. Pendse, Simulation of the dynamic behavior of deep bed filters, AIChE Journal 25 (1979) 385-395.

[20] M. Fichman, C. Gutfinger, D. Pnueli, Effect of particle loading on granular bed filtration - The cluster enhanced filter model, Journal of Aerosol Science 19 (1988) 425-441.

[21] A. Charvet, L. Wingert, N. Bardin-Monnier, S. Pacault, C. Godoy, Q. Ribeyre, D. Thomas, Visualization of airborne nanoparticle deposits onto spherical collectors, Separation and Purification Technology 172 (2017) 119129.

[22] V. Pareto, Manuale di Economia Politica. Societa Editrice Libraria, Milano. Translated into English by Ann S. Schwier, edited by Ann S. Schwier and Alfred N. Page (1971), Manual of Political Economy, Augustus M. Kelley, New York, 1906.

[23] R. Slowinski, Intelligent decision support: Handbook of Applications and Advances of the Rough Sets Theory, Kluwer Academic Publishers, Dordrecht, 1992.

[24] J. Thibault, D. Taylor, C. Yanofsky, R. Lanouette, C. Fonteix, K. Zaras, Multicriteria optimization of a high yield pulping process with rough sets, Chemical Engineering Science 58 (2003) 203-213. 